\title{
Scattering of a spherical pulse from a small inhomogeneity: Dilatation and rotation
}

\author{
M D Sharma \\ Department of Mathematics, Kurukshetra University, Kurukshetra 136 119, India
}

\begin{abstract}
Perturbations in elastic constants and density distinguish a volume inhomogeneity from its homogeneous surroundings. The equation of motion for the first order scattering is studied in the perturbed medium. The scattered waves are generated by the interaction between the primary waves and the inhomogeneity. First order scattering theory is modified to include the source term generating the primary waves. The body force equivalent to the scattering source is presented in a convenient form involving the perturbations in wave velocities and gradient of density perturbation.

A procedure is presented to study the scattering of a spherical pulse from a small inhomogeneity, in time domain. The size of inhomogeneity is assumed small as compared to its distance from source and receiver. No restrictions are placed on the positions of source, receiver and inhomogeneity. The dilatation and rotations are calculated for a pulse scattered from an arbitrary point in a spherical volume. The aggregate of the scattered phases from all the points of the inhomogeneity, reaching at a fixed receiver, gives the amount of scattering from the inhomogeneity. The interaction of both $P$ and $S$ waves with inhomogeneity are considered. Dilatation and rotations for scattering are obtained as integral expressions over the solid angle of inhomogeneity. These expressions are computed numerically, for hypothetical models. The effects of source (unit force) orientations, velocity and density perturbations, and size of inhomogeneity, on the scattered phases, are discussed.
\end{abstract}

\section{Introduction}

The inhomogeneities present in the Earth, only, can explain the various phases in a seismogram. The velocity and density heterogeneities can cause the change in waveform, phase and amplitude fluctuations. They can also generate coda (P-coda, Scoda, Lg-coda) waves and precursory waves such as the scattered PKP waves as the precursors to PKIKP waves. The delayed energy in the seismic coda is due to the waves scattering at the heterogeneities in the crust and lithosphere. In exploration geophysics, the introduction of shear wave sources and three-component geophones has multiplied the applications of elastic wave scattering to study complex objects and structural exploration. In the case of vertical seismic profiling, where source and receiver arrangements are favourable for receiving the wide-angle scattered waves, the seismic wave scattering has vast possibilities of application.

The modelling of waves scattered by a small inhomogeneity has taken a variety of approaches. The basis for any advanced scattering theory is the elastic wave scattering by a single inclusion in a homogeneous elastic medium. Korneev and Johnson (1993a,b) gave the compact solutions for single scattering by spheres of arbitrary contrast and tested them numerically. Their solutions include corrections for Mie scattering when the wavelength is comparable to the size of inhomogeneity. In a related work, Grito et al (1995) have tested the validity of the, commonly used, far-field approximation. The techniques employed so far to study seismic wave scattering problems include Maslov theory (Chapman \& Drummond

Keywords. Scattering; inhomogeneity; spherical pulse; perturbations; dilatation; rotation. 
1982), the finite difference method (Fehler and Aki 1978), the finite element method (Lysmer and Drake 1972), the Born approximation (Wu and Aki 1985a), the complex screen approach (Wu 1994), Kirchoff-Helmhotz integration (Neuberg and Pointer 1995), the boundary integral equation method (Benites, Aki and Yamogida 1992) and boundary element method (Chen and Zhou 1994). Every method has inherent advantages and disadvantages.

In the approach which best serves the purpose (Leary 1995) the wave equation can be written in terms of a uniform average medium about which the heterogeneities are local fluctuations. A first order perturbation approximation is used to express the coda wave-field in terms of scattering by a primary wavelet interacting with heterogeneities (Aki \& Richards 1980). Gubernatis et al. (1977) formulated the scattering problem of a homogeneous inclusion using Born approximation. Sato (1984) expressed Born approximation in terms of density and wave velocities in an isotropic medium. Wu and Aki (1985a,b) applied this approximation to derive scattering coefficients for elastic random media and discussed scattering characteristics of elastic waves by an arbitrary elastic heterogeneity. Wu (1989) gave the detailed analysis for weak scattering. Wu and Aki (1988) classified the short-wave scattering into different scattering phenomena, in terms of different propagation regimes characterised by wave length and scales of inhomogeneity and heterogeneous region. Rose (1989) applied elastic wave scattering theories for ultrasonic detection and characterization of flaws in metals and ceramics. To advance the scattering studies, in recent years, Gajewski (1993) studied the radiation from point sources in anisotropic media. Gurevich et al $(1992,1998)$ discussed the Born approximation and scattering in a poroelastic medium.

Most of the experiments carried out to study scattering use pulse transducer and hence data are collected in time domain. On the other hand, most of the theoretical studies on elastic wave scattering consider the incidence of plane waves. The studies considering the incidence of spherical pulse either use numerical methods or are in frequency domain (e.g., Piquette 1986, 1987; Hinders et al 1992; Korneev and Johnson 1993a,b; Tadeu et al 1996). Thus, it is a mismatch of the theory with the experiments. The fact is that the time domain study always presents a transparent scenario for any transient motion. The few analytical studies, those that consider the scattering of spherical pulse in time domain use Born approximation. The practical limitation with Born approximation is that it yields best results only for back scattering. So, the study of first order scattering of spherical pulse in the time domain without the use of Born approximation might be important in investigating wideangle scattering. Here, the author modified the scattering source representation used in the integral expression for dilatation and rotation of scattering (Aki and Richards 1980). The integral solutions are solved for the incidence of a spherical pulse, from an impulsive source, on a small spherical inhomogeneity. Arbitrary positions of source and receiver permit to study both the small and wide angle scattering.

\section{First order scattering theory}

A finite volume weak inhomogeneity is considered in an isotropic elastic homogeneous medium of Lame's moduli $\lambda_{o}, \mu_{o}$ and density $\rho_{o}$. Elastic constants and density of this inhomogeneity are defined as

$$
p=p_{o}+\delta p ; \quad(p=\lambda, \mu, \rho)
$$

where $\delta p\left(|\delta p| \ll\left|p_{o}\right|\right)$, denote the small perturbations in elastic parameters and density. The displacement in the inhomogeneous medium is written as $\mathbf{u}=\mathbf{u}^{o}+\boldsymbol{\delta} \mathbf{u}$, which is a sum of 'primary waves' $\mathbf{u}^{o}$ and 'scattered waves' $\delta \mathbf{u}$. Inhomogeneity is weak so that $|\delta \mathbf{u}| \ll\left|\mathbf{u}^{o}\right|$, and, hence, scattered waves obey the wave equations for the unperturbed medium.

The equation of motion in an inhomogeneous medium is given by

$$
\rho \ddot{u}_{i}=\rho f_{i}+\left(\lambda u_{j, j}\right)_{, i}+\left\{\mu\left(u_{i, j}+u_{j, i}\right)\right\}_{, j}
$$

where, $\mathbf{f}$ is the body force (per unit mass) representing the source fixed in the homogeneous part of the medium and generating primary waves. For a weak inhomogeneity (ignoring terms of second and higher orders of perturbations) equation (2) is resolved into two equations. These are

$$
\rho_{o} \ddot{\mathbf{u}}^{o}=\rho_{o} \mathbf{f}+\left(\lambda_{o}+\mu_{o}\right) \boldsymbol{\nabla}\left(\boldsymbol{\nabla} \cdot \mathbf{u}^{o}\right)+\mu_{o} \nabla^{2} \mathbf{u}^{o},
$$

and

$$
\delta \ddot{\mathbf{u}}=\left(\frac{\lambda_{o}+\mu_{o}}{\rho_{o}}\right) \nabla(\nabla . \delta \mathbf{u})+\left(\frac{\mu_{o}}{\rho_{o}}\right) \nabla^{2} \delta \mathbf{u}+\mathbf{Q},
$$

where,

$$
\begin{aligned}
Q_{i}= & \left(\frac{\delta \rho}{\rho_{o}}\right)\left(f_{i}-\ddot{u}_{i}^{o}\right)+\left(\frac{\delta \lambda+\delta \mu}{\rho_{o}}\right) u_{j, i j}^{o}+\left(\frac{\delta \mu}{\rho_{o}}\right) u_{i, j j}^{o} \\
& +\left(\frac{\delta \lambda_{, i}}{\rho_{o}}\right) u_{j, j}^{o}+\left(\frac{\delta \mu, j}{\rho_{o}}\right)\left(u_{i, j}^{o}+u_{j, i}^{o}\right),
\end{aligned}
$$

represents the body force (per unit mass) which is determined by the interaction of inhomogeneity 


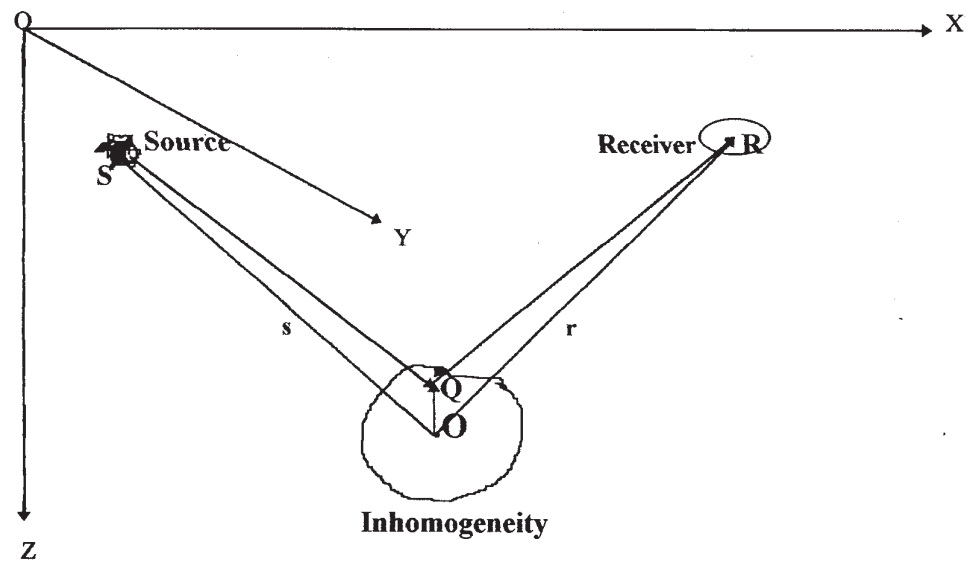

Figure 1. Geometry of the medium.

and primary waves from source. Equation (3) is the equation of motion for the homogeneous surrounding of small inhomogeneity and equation (4) represents the incremental motion due to the presence of inhomogeneity in a homogeneous medium (Aki and Richards 1980). Body force term (i.e., $\left.\rho_{o} \mathbf{f}\right)$ in equation (3) represents the source which is generating primary waves. $\mathbf{Q}$, given by (5), is a body force equivalent of the scattering source. This expression of $\mathbf{Q}$ also considers the direct contribution of original source, i.e., body force $\mathbf{f}$, to scattering.

Using (3) in (5), changing the elastic parameter perturbations to velocity perturbations and ignoring the terms of second and higher orders of perturbations, we can write

$$
\begin{aligned}
Q_{i}= & \left(2 \alpha_{o} \delta \alpha\right) u_{j, i j}^{o}+\left(2 \alpha_{o} \delta \alpha+\alpha_{o}^{2} \frac{\delta \rho}{\rho_{o}}\right)_{, i} u_{j, j}^{o} \\
+ & \left(2 \beta_{o} \delta \beta\right)\left(u_{i, j j}^{o}-u_{j, i j}^{o}\right)+\left(2 \beta_{o} \delta \beta+\beta_{o}^{2} \frac{\delta \rho}{\rho_{o}}\right)_{, j} \\
& \left(u_{i, j}^{o}+u_{j, i}^{o}-2 \delta_{i j} u_{k, k}^{o}\right) .
\end{aligned}
$$

The above expression of $\mathbf{Q}$ is quite different from the one being used earlier (Aki and Richards 1980). The term representing the single force (i.e., $\rho_{o} \ddot{\mathbf{u}}^{o}$ ) is absent. Also, not the density perturbation but its gradient does affect the scattering directly and, hence, the smooth variations of the density do not contribute to the first order scattering.

From (4) we can write

$$
\begin{gathered}
\frac{\partial^{2}(\boldsymbol{\nabla} . \boldsymbol{\delta} \mathbf{u})}{\partial t^{2}}=\alpha_{o}^{2} \nabla^{2}(\boldsymbol{\nabla} . \boldsymbol{\delta} \mathbf{u})+\boldsymbol{\nabla} . \mathbf{Q}, \\
\frac{\partial^{2}(\boldsymbol{\nabla} \wedge \boldsymbol{\delta} \mathbf{u})}{\partial t^{2}}=\beta_{o}^{2} \nabla^{2}(\nabla \wedge \boldsymbol{\delta} \mathbf{u})+\nabla \wedge \mathbf{Q} .
\end{gathered}
$$

Solutions of (7) and (8), for waves scattered from a small volume $V$ and recorded at $\mathbf{r}$, can be written as

$$
\begin{aligned}
\nabla . \delta \mathbf{u}(\mathbf{r}, t)= & \frac{1}{4 \pi \alpha_{o}^{2}} \int \frac{1}{|\mathbf{r}-\mathbf{q}|} \nabla \cdot \mathbf{Q}\left(\mathbf{q}, t-\frac{|\mathbf{r}-\mathbf{q}|}{\alpha_{o}}\right) \\
& \mathrm{d} V(\mathbf{q})
\end{aligned}
$$

and

$$
\begin{aligned}
\nabla \wedge \boldsymbol{\delta} \mathbf{u}(\mathbf{r}, t)= & \frac{1}{4 \pi \beta_{o}^{2}} \int \frac{1}{|\mathbf{r}-\mathbf{q}|} \nabla \wedge \mathbf{Q}\left(\mathbf{q}, t-\frac{|\mathbf{r}-\mathbf{q}|}{\beta_{o}}\right) \\
& \mathrm{d} V(\mathbf{q}) .
\end{aligned}
$$

$V$ is the inhomogeneous region where $\mathbf{Q}$ is nonzero.

\section{Geometry of the medium}

Consider a cartesian coordinate system $(x, y, z)$ with source at $S \leftrightarrow\left(S_{x}, S_{y}, S_{z}\right)$ (figure 1). The unit vector $\mathbf{n}=\left(n_{x}, n_{y}, n_{z}\right)$ defines the direction of the force at $S$. Centre of the inhomogeneity is represented by $O \leftrightarrow\left(O_{x}, O_{y}, O_{z}\right)$. An arbitrary location for receiver is represented by $R \leftrightarrow\left(R_{x}, R_{y}, R_{z}\right)$. Hence we have $\mathbf{s}=\left(S_{x}-O_{x}, S_{y}-O_{y}, S_{z}-O_{z}\right)$ and $\mathbf{r}=\left(R_{x}-O_{x}, R_{y}-O_{y}, R_{z}-O_{z}\right)$. For an arbitrary point $Q \leftrightarrow\left(Q_{x}, Q_{y}, Q_{z}\right)$ in the inhomogeneity the variable $\mathbf{q}=\left(Q_{x}-O_{x}, Q_{y}-O_{y}, Q_{z}-O_{z}\right)$. In a local spherical coordinate system centered at $O$ with $\mathbf{s}$ being its polar axis, the variable $\mathbf{q}=$ $(q, \theta, \phi)$ related to its cartesian components by $q_{x}=$ $q \sqrt{1-\chi_{s}^{2}} \cos \phi, q_{y}=q \sqrt{1-\chi_{s}^{2}} \sin \phi$ and $q_{z}=q \chi_{s}$. $\chi_{s}(=\cos \theta)$ varies from -1 to 1 and $\phi$ varies from 0 to $2 \pi$. For a spherical inhomogeneity $q$ will be varying over its radius and $\mathrm{d} V=q^{2} \mathrm{~d} q \mathrm{~d} \Omega ; \mathrm{d} \Omega=\mathrm{d} \chi_{s} \mathrm{~d} \phi$.

\section{Scattering of an impulse}

The source at $S$ is an impulsive point unit force in the direction $\left(n_{x}, n_{y}, n_{z}\right)$. The radially polarised 
spike wavefield, given by,

$$
\mathbf{u}^{o}(\mathbf{q}, t)=4 \pi u_{o} \mathbf{d} \frac{s_{o}}{|\mathbf{q}-\mathbf{s}|} \delta\left(t-\frac{|\mathbf{q}-\mathbf{s}|}{v_{I}}\right)
$$

represents the displacement at a point $\mathbf{q}$. This is a far-field solution of equation (3) in the presence of body force $\mathbf{f}$ as a source at $S$. The normalisation of (11) is the displacement $4 \pi u_{o}$ at a distance $s_{o}$ from the source and at time $t=s_{o} / v_{I} . v_{I}$ is the velocity of the waves radiating from the source, i.e., $\alpha_{o}$ for $P$ wave and $\beta_{o}$ for $S$ wave. The polarisation vector $\mathbf{d}$ is given by: $d_{i}=\gamma_{i} \gamma_{j} n_{j}$ for $P$ wave; and $d_{i}=\left(\delta_{i j}-\gamma_{i} \gamma_{j}\right) n_{j}$ for $S$ wave. The direction cosines $\gamma_{j}=\left(q_{j}-s_{j}\right) /|\mathbf{q}-\mathbf{s}| ;(j=x, y, z)$, control the angular dependence of point force radiation. Time $^{-1}$ dimension of $\delta$ function in $\mathbf{u}^{o}$ is consumed on its convolution with time dependent force.

The spherical pulse given by (11), acts as a primary wave which after interaction with inhomogeneity generates a scattering source in the form of body force $\mathbf{Q}$. The waves from this scattering source have their dilatation and rotation defined by the integral expressions (9) and (10), respectively. These expressions are, then, solved using the following assumptions.

- $q \ll s$, and hence $|\mathbf{s}-\mathbf{q}| \approx s\left(1-q \chi_{s} / s\right)$.

- $q \ll r$, and hence $|\mathbf{r}-\mathbf{q}| \approx r\left(1-q \chi_{r} / r\right),\left(\chi_{r}\right.$ is cosine of angle between $\mathbf{q}$ and $\mathbf{r}$ ).

- The spherically symmetric perturbations, represented by $F\left(=\frac{\delta \alpha}{\alpha_{o}}\right), G\left(=\frac{\delta \beta}{\beta_{o}}\right)$, and $H\left(=\frac{\delta \rho}{\rho_{o}}\right)$, are twice differentiable functions of $q$.

- The terms with more than third order of $r$ and $s$ together in denominator are ignored.

- The inhomogeneity is a spherical volume so that $\mathrm{d} V=q^{2} \mathrm{~d} q \mathrm{~d} \Omega$.

- $\delta$ function and its derivatives vanish at the limits of integration over $q$.

After a lengthy calculation which contains many differentiation and integration with $q$, the incremental dilatation $(\boldsymbol{\nabla} . \boldsymbol{\delta} \mathbf{u})$ from equation (9), is given by

$$
\begin{aligned}
\nabla . \delta \mathbf{u}= & u_{o} s_{o}\left[\frac{1}{r s} \int v_{1} D_{1} \mathrm{~d} \Omega+\frac{1}{r^{2} s} \int \chi_{r} v_{1} D_{2} \mathrm{~d} \Omega\right. \\
& \left.+\frac{1}{r s^{2}} \int v_{1} D_{3} \mathrm{~d} \Omega\right]
\end{aligned}
$$

where,

$$
\begin{aligned}
v_{1} & =\left(\frac{\chi_{r}}{\alpha_{o}}+\frac{\chi_{s}}{v_{I}}\right)^{-1} \\
D_{1} & =2 a\left[2 F_{o}^{\prime}+\frac{\beta_{o}^{2}}{\alpha_{o}^{2}}\left(q_{o} H_{o}^{\prime \prime}+H_{o}^{\prime}\right)\right],
\end{aligned}
$$

$$
\begin{aligned}
D_{2}= & a\left(1+2 \frac{\beta_{o}^{2}}{\alpha_{o}^{2}}\right)\left(q_{o}^{2} H_{o}^{\prime \prime}+2 q_{o} H_{o}^{\prime}\right), \\
D_{3}= & (3 b-c)\left[4\left(q_{o} F_{o}^{\prime}+F_{o}\right)-4 \frac{\beta_{o}^{2}}{\alpha_{o}^{2}}\left(q_{o} G_{o}^{\prime}\right)\right. \\
& \left.+\frac{\beta_{o}^{2}}{\alpha_{o}^{2}}\left(q_{o}^{2} H_{o}^{\prime \prime}\right)\right],
\end{aligned}
$$

for $q_{o}=-\left(t-\frac{r}{\alpha_{o}}-\frac{s}{v_{I}}\right) v_{1}$.

Similarly, the incremental rotation $(\boldsymbol{\nabla} \wedge \boldsymbol{\delta} \mathbf{u})$, from equation (10), is given by

$$
\begin{aligned}
\boldsymbol{\nabla} \wedge \boldsymbol{\delta} \mathbf{u}= & u_{o} s_{o}\left[\frac{1}{r s} \int v_{2} \boldsymbol{\Omega}_{\mathbf{1}} \mathrm{d} \Omega+\frac{1}{r^{2} s} \int \chi_{r} v_{2} \boldsymbol{\Omega}_{\mathbf{2}} \mathrm{d} \Omega\right. \\
& \left.+\frac{1}{r s^{2}} \int v_{2} \boldsymbol{\Omega}_{\mathbf{3}} \mathrm{d} \Omega\right],
\end{aligned}
$$

where,

$$
\begin{aligned}
v_{2}= & \left(\frac{\chi_{r}}{\beta_{o}}+\frac{\chi_{s}}{v_{I}}\right)^{-1} \\
\boldsymbol{\Omega}_{\mathbf{1}}= & \mathbf{A}\left[4 G_{o}^{\prime}+\left(1+\frac{\alpha_{o}^{2}}{\beta_{o}^{2}}\right)\left(q_{o} H_{o}^{\prime \prime}+H_{o}^{\prime}\right)\right] \\
\boldsymbol{\Omega}_{\mathbf{2}}= & \mathbf{A}\left(2+\frac{\alpha_{o}^{2}}{\beta_{o}^{2}}\right)\left(q_{o}^{2} H_{o}^{\prime \prime}+2 q_{o} H_{o}^{\prime}\right), \\
\boldsymbol{\Omega}_{\mathbf{3}}= & 2\left[(3 \mathbf{B}-2 \mathbf{C}-3 \mathbf{D})\left(q_{o} G_{o}^{\prime}+G_{o}\right)+3(\mathbf{B}+\right. \\
& \left.\mathbf{D}) G_{o}\right]+\left[(\mathbf{B}+\mathbf{D}) \frac{\alpha_{o}^{2}}{\beta_{o}^{2}}+(2 \mathbf{B}-\mathbf{C}-\mathbf{D})\right] \\
& \left(q_{o}^{2} H_{o}^{\prime \prime}+2 q_{o} H_{o}^{\prime}\right)-3(\mathbf{B}+\mathbf{D}) q_{o} H_{o}^{\prime},
\end{aligned}
$$

for, $q_{o}=-\left(t-\frac{r}{\beta_{o}}-\frac{s}{v_{I}}\right) v_{2}$.

Subscript ' $O$ ' denotes the value of a function (or its derivatives) at $q=q_{o}$. For the evaluation of $D_{j}$ and $\boldsymbol{\Omega}_{j}, q_{o}$ is restricted to be lying in the inhomogeneity (i.e., for a spherical inhomogeneity of radius $\xi$ (say), at a given time $t$, any point in the inhomogeneity giving $q_{o}$ outside $[0, \xi]$, does not contribute to the scattering).

Other parameters used in equations (12)-(21) are defined as follows.

For incident $P$ wave :

$$
\begin{aligned}
a & =\chi_{s} \chi_{o} ; \quad b=\left(3 \chi_{s}^{2}-1\right) \chi_{o}-\chi_{s} \chi_{f} ; \quad c=-\chi_{o} ; \\
\mathbf{A} & =\chi_{o} \boldsymbol{\Psi}_{s} ; \quad \mathbf{B}=\left(3 \chi_{o} \chi_{s}-\chi_{f}\right) \boldsymbol{\Psi}_{s} ; \quad \mathbf{C}=\boldsymbol{\Psi}_{o} ; \\
\mathbf{D} & =\left(3 \chi_{o} \boldsymbol{\Psi}_{s}-\boldsymbol{\Psi}_{f}\right) \chi_{s} .
\end{aligned}
$$

For incident $S$ wave :

$$
\begin{aligned}
a= & \chi_{f}-\chi_{s} \chi_{o} ; \quad b=2 \chi_{s} \chi_{f}-\left(3 \chi_{s}^{2}-1\right) \chi_{o} ; \\
c= & 2 \chi_{o} ; \\
\mathbf{A}= & \boldsymbol{\Psi}_{f}-\chi_{o} \boldsymbol{\Psi}_{s} ; \quad \mathbf{B}=\mathbf{D}=\chi_{s} \boldsymbol{\Psi}_{f}-\left(3 \chi_{o} \chi_{s}\right. \\
& \left.-\chi_{f}\right) \boldsymbol{\Psi}_{s} ; \quad \mathbf{C}=\mathbf{0} .
\end{aligned}
$$

where,

$$
\begin{aligned}
& \chi_{o}=\hat{\mathbf{n}} . \hat{\mathbf{s}}, \quad \chi_{f}=\hat{\mathbf{n}} \cdot \hat{\mathbf{q}} \quad \chi_{s}=\hat{\mathbf{s}} \cdot \hat{\mathbf{q}} ; \\
& \boldsymbol{\Psi}_{o}=\hat{\mathbf{n}} \wedge \hat{\mathbf{s}} ; \quad \boldsymbol{\Psi}_{f}=\hat{\mathbf{n}} \wedge \hat{\mathbf{q}} ; \quad \boldsymbol{\Psi}_{s}=\hat{\mathbf{s}} \wedge \hat{\mathbf{q}} .
\end{aligned}
$$




\section{Special Cases}

(a) Assuming density perturbation as a constant may be a little compromise but it simplifies the expressions to a great extent. So, when the derivatives of $H(q)$ vanish, the expressions (14)-(16) and (19)-(21) reduce as follows.

$$
\begin{aligned}
D_{1} & =4 a F_{o}^{\prime}, \\
D_{2} & =0, \\
D_{3} & =4(3 b-c)\left[\left(q_{o} F_{o}^{\prime}+F_{o}\right)-\frac{\beta_{o}^{2}}{\alpha_{o}^{2}}\left(q_{o} G_{o}^{\prime}\right)\right], \\
\boldsymbol{\Omega}_{1} & =4 \mathbf{A} G_{o}^{\prime}, \\
\boldsymbol{\Omega}_{\mathbf{2}} & =\mathbf{0}, \\
\boldsymbol{\Omega}_{\mathbf{3}} & =2\left[(3 \mathbf{B}-2 \mathbf{C}-3 \mathbf{D})\left(q_{o} G_{o}^{\prime}+G_{o}\right)+3\right. \\
& \left.(\mathbf{B}+\mathbf{D}) G_{o}\right] .
\end{aligned}
$$

It may be noted that major contributions to scattering come from $D_{1}$ and $\Omega_{1}$ and these terms are containing the gradient of functions $F(q)$ and $G(q)$ respectively. These results improve upon and generalise the results of Leary (1995) showing the scattering of SHmotion proportional to the velocity gradient.

(b) For a smooth inhomogeneity, spatial gradients of elastic parameters vanish. Then the dilatation and rotation of scattering are given by,

$$
\begin{aligned}
\nabla . \delta \mathbf{u} & =u_{o} s_{o}\left[\frac{4}{r s^{2}} \frac{\delta \alpha}{\alpha_{o}} \int v_{1}(3 b-c) \mathrm{d} \Omega\right], \\
\nabla \wedge \boldsymbol{\delta} \mathbf{u} & =u_{o} s_{o}\left[\frac{4}{r s^{2}} \frac{\delta \beta}{\beta_{o}} \int v_{2}(3 \mathbf{B}-\mathbf{C}) \mathrm{d} \Omega\right] .
\end{aligned}
$$

The above expressions have $r s^{2}$ in the denominator which means that dilatation and rotation in this case will be very small as compared to the case (a).

(c) Back scattering results can be obtained by keeping the positions of both receiver and source nearby. Also, $r \approx s$ and $\chi_{r} \approx \chi_{s}$.

(d) From a point scatterer, at $\left(q_{o}=0\right)$, we have

$$
\begin{aligned}
\boldsymbol{\nabla} . \boldsymbol{\delta} \mathbf{u}= & u_{o} s_{o}\left[\frac{2}{r s}\left\{2 F^{\prime}(0)+\frac{\beta_{o}^{2}}{\alpha_{o}^{2}} H^{\prime}(0)\right\} \int v_{1} a \mathrm{~d} \Omega\right. \\
& \left.+\frac{4}{r s^{2}} \int v_{1}(3 b-c) \mathrm{d} \Omega\right] \\
\nabla \wedge \boldsymbol{\delta} \mathbf{u}= & u_{o} s_{o}\left[\frac{1}{r s}\left\{4 G^{\prime}(0)+\left(1+\frac{\alpha_{o}^{2}}{\beta_{o}^{2}}\right) H^{\prime}(0)\right\}\right. \\
& \left.\int v_{2} \mathbf{A} \mathrm{d} \Omega+\frac{4}{r s^{2}} G(0) \int v_{2}(3 \mathbf{B}-\mathbf{C}) \mathrm{d} \Omega\right]
\end{aligned}
$$

(e) The dilatation and rotation are assumed constant in the spherical inhomogeneity of radius $\xi$. Then, using Gauss' divergence theorem and Stokes' theorem, displacements $\delta u^{(P)}$ and $\delta \mathbf{u}^{(S)}$ for $P$ wave and $S$ wave, respectively, are expressed as $\delta u^{(P)}=\frac{\xi}{3} \nabla . \delta \mathbf{u}, \delta \mathbf{u}^{(S)}=\frac{\xi}{2} \boldsymbol{\nabla} \wedge \boldsymbol{\delta} \mathbf{u}$.

\section{Numerical results}

The purpose of numerical calculation is to compute the integral expressions (12)-(21). Density perturbations are assumed constant to simplify the computation. Perturbations in wave velocities are defined by $F(q)=G(q)=0.05 \exp (-k q)$, where $k$ represents the rate of exponential decay of radial inhomogeneity of velocities. $k$ may be called velocity perturbation parameter. Locations of source, receiver and inhomogeneity, in meters' scale are chosen as $S \leftrightarrow(0,0,240) ; R \leftrightarrow(75,0,230) ; O \leftrightarrow$ $(35,15,250)$. Wave velocities are assumed as $\alpha_{o}=$ $2.5 \mathrm{~km} / \mathrm{sec} ; \beta_{o}=1.4 \mathrm{~km} / \mathrm{sec}$. Integral expressions are solved by summation over $\left(\chi_{s}, \phi\right)$ plane.

For qualitative purposes, the dilatation may represent $P$ wave and the rotation may represent $S$ wave. Therefore, dilatation and rotation of scattering due to the incidence of $P$ wave are identified as PP phase and PS phase, respectively. Similarly, SP and SS phases are identified for the $S$ wave incidence.

In order to study the effects of the size of inhomogeneity $(\xi)$, perturbation parameter $(k)$ and source orientation $(\mathbf{n})$, various phases were computed and some of them are exhibited in figures 2 to 4 . Details are as follows.

PP phase and $y$-component of SS phase are plotted, in figure 2 , for values of $\xi=5,8,12$ meters. It is observed that the width of scattered pulse increases with the increase in the size of inhomogeneity. Effect on magnitude of the phase is not much. SP phase and z-component of PS phase are plotted, in figure 3 , for the values of velocity perturbation parameter $k=0.02,0.05,0.1$. Magnitude of scattering decreases with the increase in rate of velocity decay inside the inhomogeneity. Width of scattered pulse is unaffected with the change of $k$. Figure 4 contains all the scattered phases for the direction of unit force along coordinate axes. Dilatations (PP and SP phases) are affected most by the component of force along $z$-axis. Rotation due to $P$ wave incidence is dominated by $x$-component of force. SS phase is a mixed contribution of all the components of force, but $y$-component is more dominating.

Figure 5 represents synthetic seismograms as recorded at a vertical borehole array. It contains the dilatation and $z$-components of rotation of the scattering from a spherical inhomogeneity of $10 \mathrm{~m}$ radius centered at $(20,10,235) \mathrm{m}$. Source is a unit impulse, at $(0,0,240 \mathrm{~m})$ along $z$-axis. Perturbations parameter $k=0.1$. Receivers are placed at depths between $220 \mathrm{~m}$ and $250 \mathrm{~m}$ at regular intervals of $5 \mathrm{~m}$. 

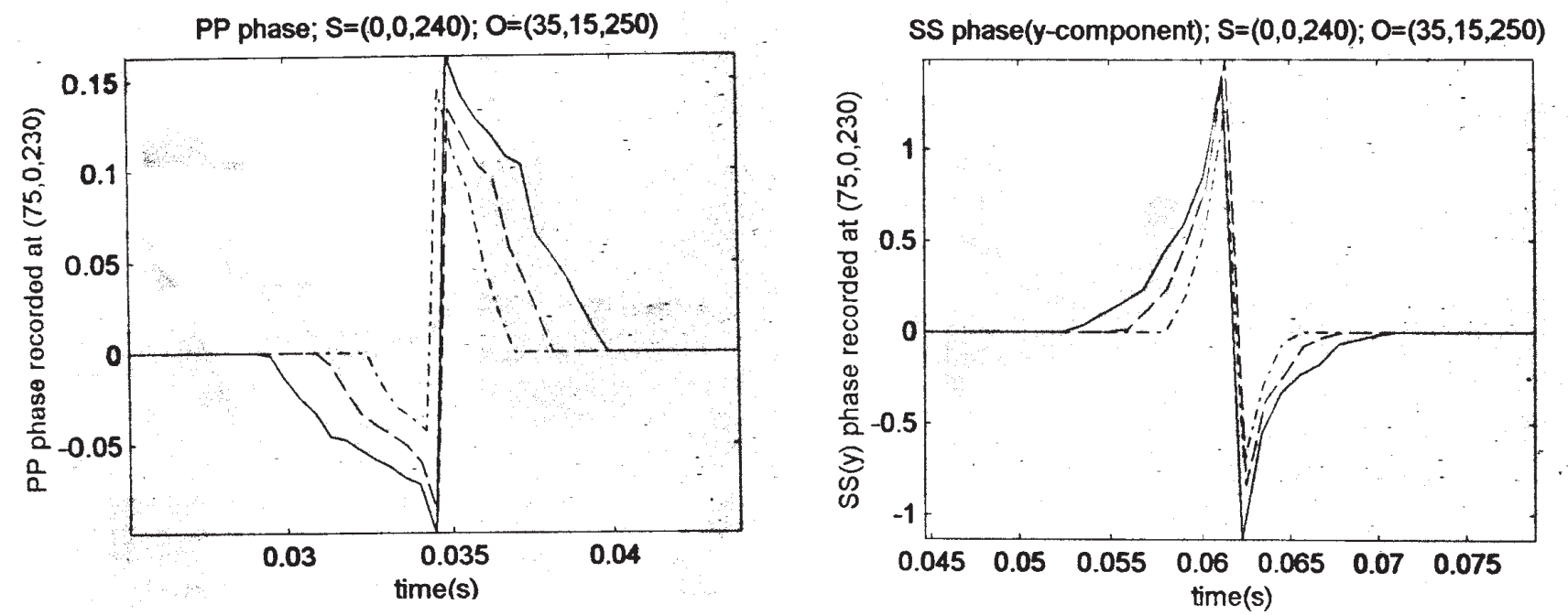

Figure 2. Variations in scattering with the size of inhomogeneity $(k=.05 ; \mathbf{n}=(1,0,0))(\xi=5 \mathrm{~m}:-\cdot-\cdot-\cdot \cdot-$ $\xi=8 \mathrm{~m}:-----\xi=12 \mathrm{~m}:-)$.
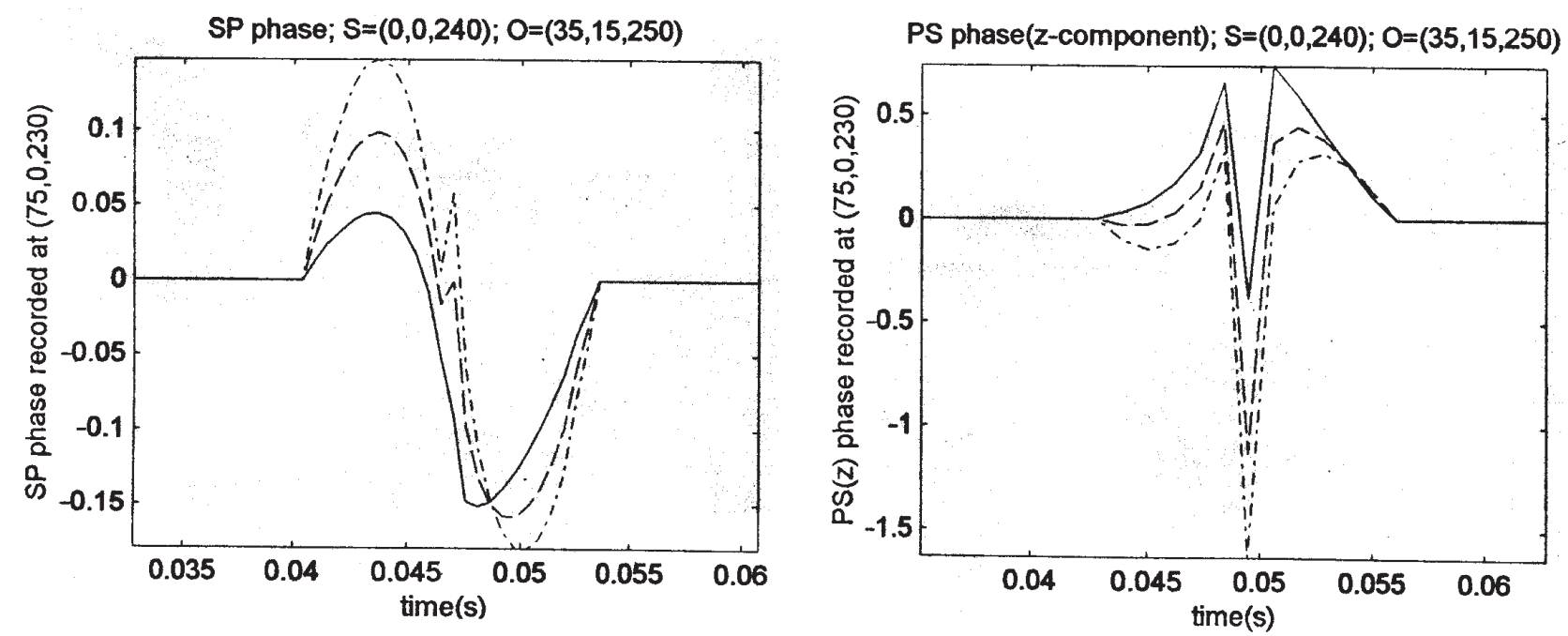

Figure 3. Variations in scattering with the velocity perturbation $(\mathbf{n}=(1,0,0) ; \xi=10 \mathrm{~m})(k=.02:-\cdot-\cdot \cdot-\cdot-$ $k=.05:------\quad k=.1: \longrightarrow$ ).

\section{Discussion of results}

This was an attempt to study the 3-D scattering from a spherical inhomogeneity in time domain. No compromise has been made for the locations of source and receiver (i.e. small- or wide-angle scattering). Velocity and density perturbations are represented by arbitrary functions. In this study, scattering source is represented in terms of velocity inhomogeneities and gradient of density inhomogeneity. The integrals for the dilatation and rotation of scattering are solved to study the role of inhomogeneity parameters. It is found that rotation of scattering doesn't involve the function $F(q)$ and its derivatives. The dilatation expression contains the gradient of function $F(q)$ but in less important part, $\left(D_{3}\right)$. The radius (size) of inho- mogeneity, i.e., $\xi$ is not involved in the scattering expressions but, for a given value of time $t$, it restricts the values of solid angle $\Omega$ to get the value of $q_{o}$ between 0 and $\xi$. This seems to be an important result for spherical wave scattering in the time domain. Also, the scattering of spherical pulse discussed in Leary (1995) is not related to the size of inhomogeneity. The main contributions to scattering come from the terms containing velocity gradients. This implies that scattering is directly related to the velocity perturbation parameter, i.e., $k$. The increase of the exponential decay of inhomogeneity decreases the scattering (figure 3). The magnitudes of dilatation and all the components of rotation changes significantly with the direction of the impulsive force (figure 4). 

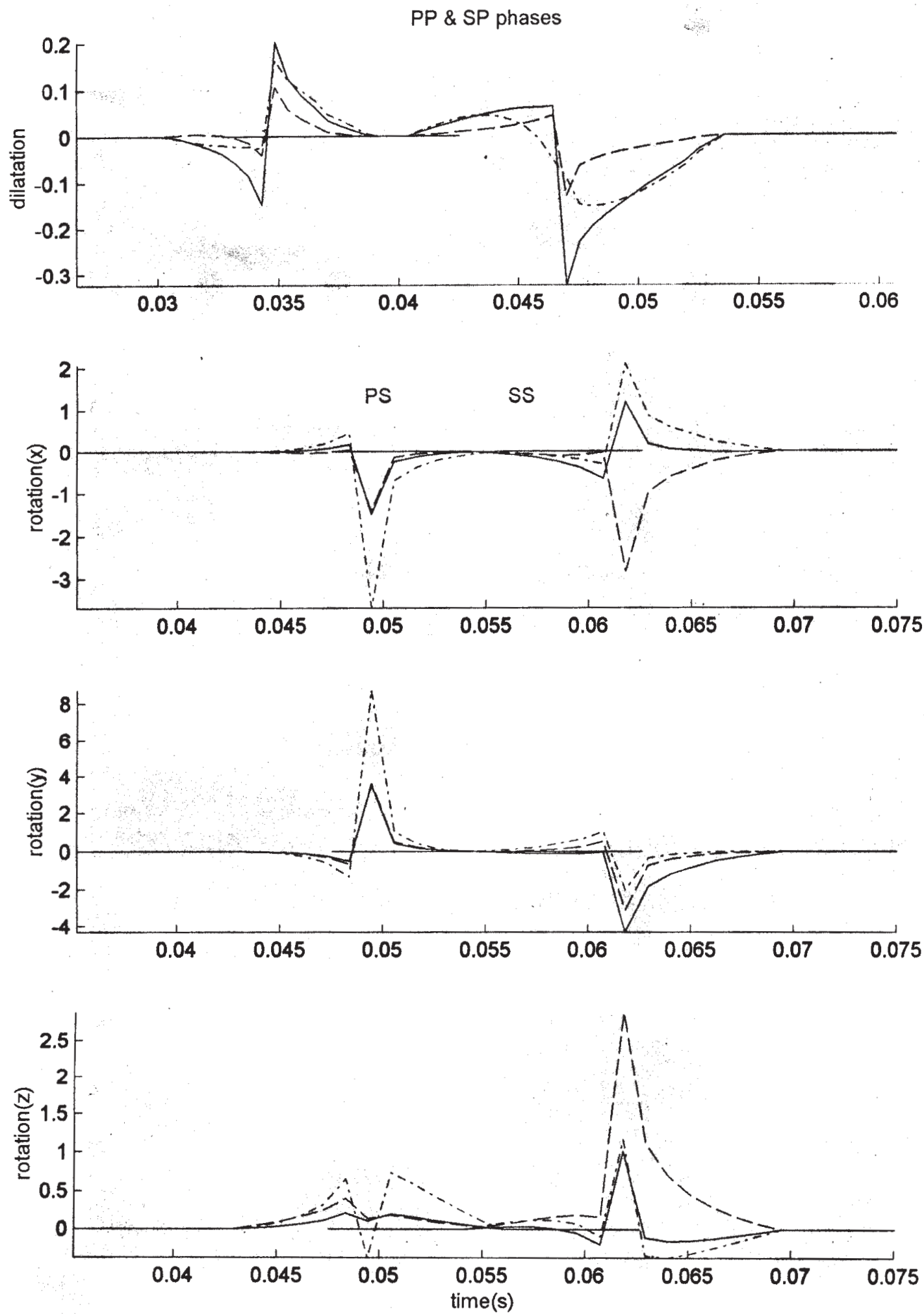

Figure 4. Variations in scattering with the direction of impulsive force $\{k=.1 ; \xi=10 \mathrm{~m}\}\{\mathbf{n}=(1,0,0):-\cdot-\cdot-\cdot-\cdot-$ $\mathbf{n}=(0,1,0):-----\mathbf{n}=(0,0,1):-\}$. 

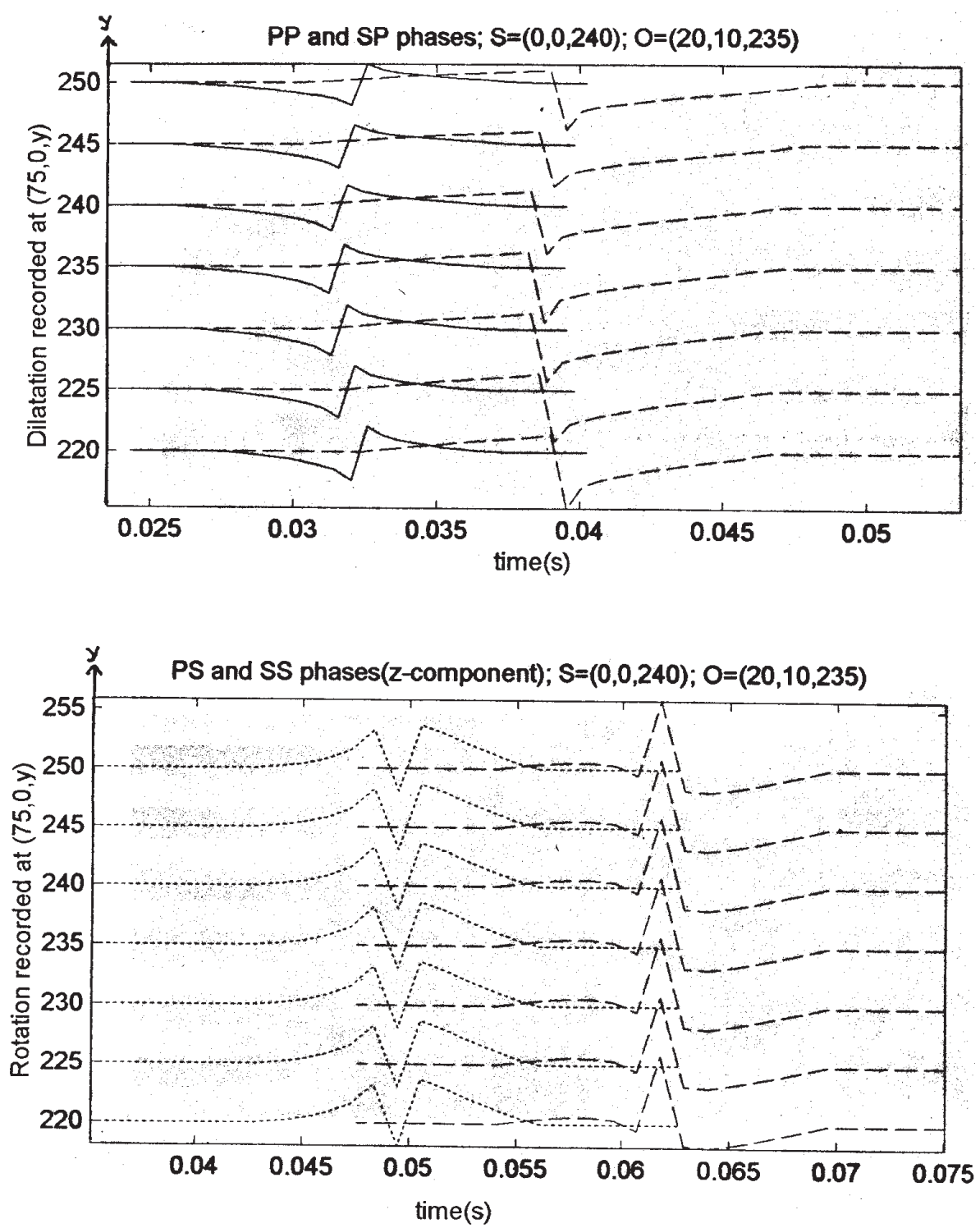

Figure 5. Scattered phases recorded at vertical borehole array.

The immediate application of the study presented may be in interpretation and simulation of the seismograms recorded at a borehole array (figure 5) during a uniwell or crosswell survey, for example, Paulsson et al (1994). The time-lapse difference in seismograms (from repeated sources) may indicate the changes in the location of scatterer around an oil-producing well. The location of the scatterer can be estimated by modelling the travel times of various scattered phases. Pulse width of scattered phases may help to estimate the size of the inhomogeneity. The expressions derived could not be verified practically due to the nonavailability of the relevant data.

\section{Conclusions}

From the analytical expressions, special cases and discussion of results, the following conclusions may be drawn.
- Smoothly varying density doesn't affect the first order scattering.

- In a far-field, a smooth inhomogeneity produces a very small scattering.

- $S$ wave velocity inhomogeneity has very little effect on the dilatation of scattering.

- $P$ wave velocity inhomogeneity has no effect on the rotation of scattering.

- Inhomogeneity contrast has a more dominating effect on the magnitude of dilatation and rotation of scattered waves, as compared to the size of inhomogeneity. This may be due to the smaller size of inhomogeneity as compared to its distance from source and receiver.

In addition to the results mentioned above it is noted that the pulse width of the scattered phases is decided by the size of inhomogeneity. The direction of the impulsive force at the source may control the magnitudes and directions of scattered phases at a fixed receiver. 


\section{Acknowledgements}

The author is thankful to the Commonwealth Scholarship Commission (U.K.) for the award of Commonwealth Fellowship to work in the University of Edinburgh. Assistance from Dr. Peter Leary and Prof. Stuart Crampin (Dept. of Geology \& Geophysics, University of Edinburgh) is gratefully acknowledged. The author is indebted to the reviewer for valuable suggestions for improving the manuscript.

\section{References}

Aki K and Richards P G 1980 Quantitative seismology, theory and methods, (New York: Freeman)

Benites R, Aki K and Yomogida K 1992 Multiple scattering of SH waves in 2-D media with many cavities; PAGEOPH $138353-390$

Chapmann C H and Drummond R 1982 Body-wave seismograms in inhomogeneous media using Maslov asymptotic theory; Bull. Seism. Soc. Am. 72 277-317

Chen G and Zhou H 1994 Boundary element modeling of nondispersive and dispersive waves; Geophysics 59 113118

Fehler M and Aki K 1978 Numerical study of diffraction of plane elastic waves by a finite crack with application to location of magma lens; Bull. Seism. Soc. Am. 68 573598

Gajewski D 1993 Radiation from point sources in general anisotropic media; Geophys. J. Int. 113 299-317

Gritto R, Korneev V A and Johnson L R 1995 Low frequency elastic wave scattering by an inclusion: limits of applications; Geophys. J. Int. 120 677-692

Gubernatis J E, Domany E, Krumhansl J A and Huberman M 1977 The Born approximation of the theory of scattering of ultrasound by flaws in elastic materials; $J$. Appl. Phys. 48 2812-2819

Gurevich B, Sadovnichaja A P, Lopatnikov S L and Shapiro S A 1992 The Born approximation in the problem of elastic wave scattering by a spherical inhomogeneity in a fluid saturated porous media; Appl. Phys. Lett. 61 1275-1277

Gurevich B, Sadovnichaja A P, Lopatnikov S L and Shapiro S A 1998 Scattering of a compressional wave in a poroelastic medium by an ellipsoidal inclusion; Geophys. J. Int. 133 91-103
Hinders M, Bogan S D and Fang T 1992 Spherical wave scattering from an elastic sphere; Il Nuovo Cimento, 107B \#10, 1215

Korneev V A and Johnson L R 1993a Scattering of elastic waves by a spherical inclusion-I: Theory and numerical results; Geophys. J. Int. 115 230-250.

Korneev V A and Johnson L R 1993b Scattering of elastic waves by a spherical inclusion-II: Limitations of asymptotic solutions; Geophys. J. Int. 115 251-263

Leary P C 1995 Quantifying crustal fracture heterogeneity by seismic scattering; Geophys. J. Int. 122 125-142

Lysmer J and Drake L A 1972 A finite element method for seismology, Methods of Computational Physics. Vol.11. (New York: Academic Press)

Neuberg J and Pointer T 1995 Modelling seismic reflections from $D^{\prime \prime}$ using the Kirchoff method; Phys. Earth Planet. Inter. $90273-281$

Piquette P C 1986 Spherical wave scattering by an elastic solid cylinder - A numerical comparison of an approximate theory with the exact theory; J. Acoust. Soc. Am. 82 699-702

Piquette P C 1987 Spherical wave scattering by an elastic solid cylinder of infinite length; J. Acoust. Soc. Am. $\mathbf{7 9}$ $1248-59$

Paulsson B N P, Meredith J A, Wang Z and Fairborn J W 1994 The Steepbank crosswell seismic project: Reservoir definition and evaluation of steam flood technology in Alberta tar sands; The Leading Edge $\mathbf{1 3}$ $737-747$

Rose J H 1989 Elastic wave inverse scattering in nondestructive evaluation; PAGEOPH 131 715-739

Sato H 1984 Attenuation and envelope formation of three component seismograms of small local earthquakes in randomly inhomogeneous lithosphere; J. Geophys. Res. 89 1221-1242

Tadeu A J B, Kausel E and Vrettos C 1996 Scattering of waves by subterranean structures via the boundary element method; Soil Dynamics and Earthq. Engg. 15 387397

Wu R S 1989 The perturbation method in elastic wave scattering; PAGEOPH 131 605-637

Wu R S 1994 Wide angle elastic wave one-way propagation in heterogeneous media and an elastic wave complexscreen method; J. Geophys. Res. 99 751-766

Wu R S and Aki K 1985a Scattering characteristics of elastic waves by an elastic heterogeneity; Geophysics $\mathbf{5 0}$ $582-595$

Wu R S and Aki K 1985b Elastic wave scattering by a random medium and the small scale inhomogeneities in the lithosphere; J. Geophys. Res. 90 10261-10273

Wu R S and Aki K 1988 Introduction: Seismic wave scattering in three-dimensionally heterogeneous earth; PAGEOPH 128 1-6 